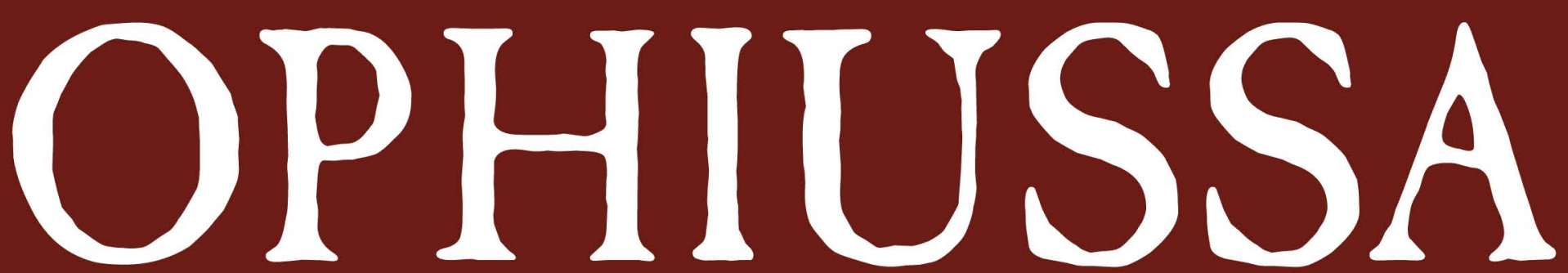

REVISTA DO CENTRO DE ARQUEOLOGIA DA UNIVERSIDADE DE LISBOA

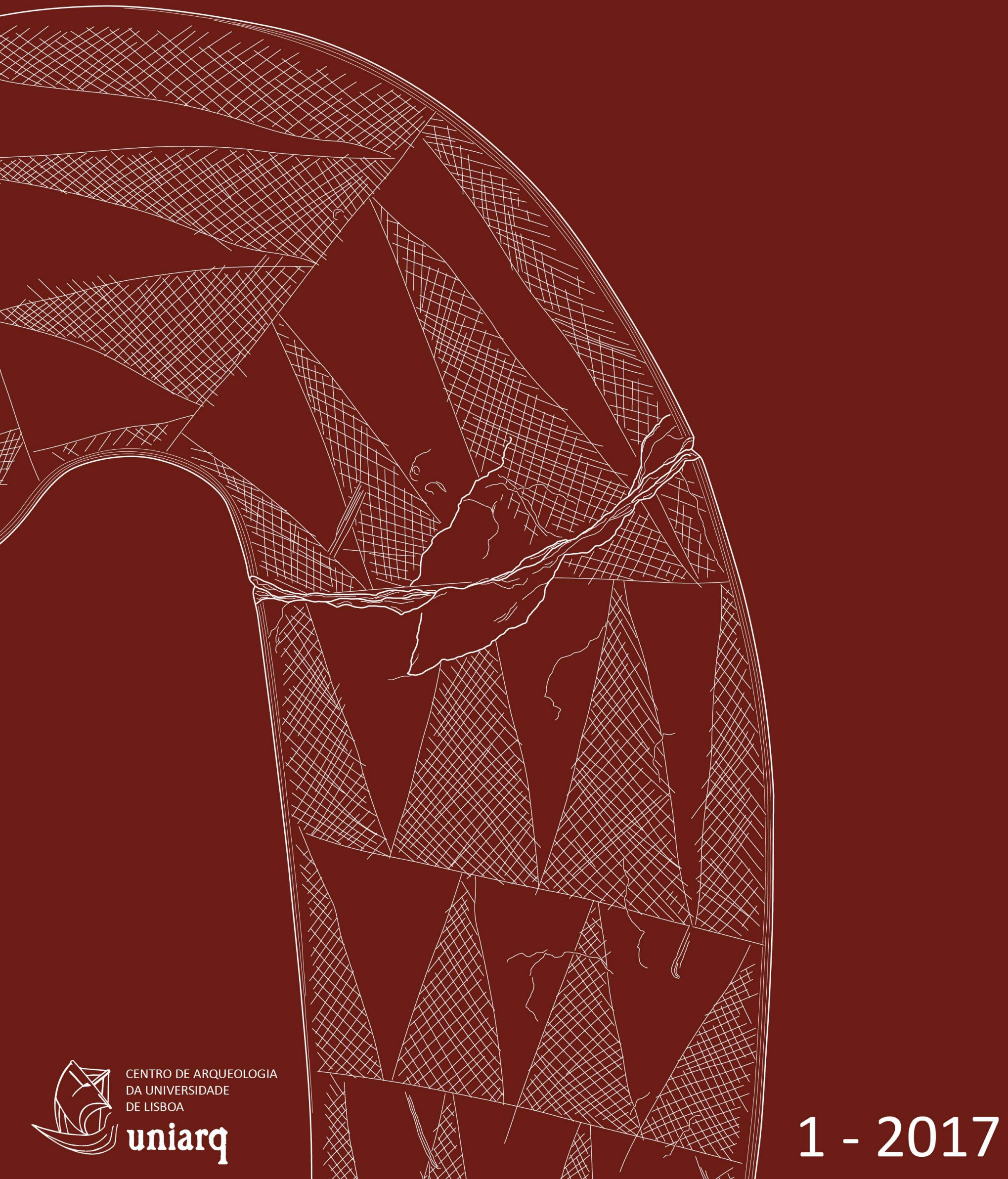


OPHIUSSA. Revista do Centro de Arqueologia da Universidade de Lisboa

ISSN $1645-653 X$

Publicação anual

Volume 1 - 2017

Direcção e Coordenação Editorial:

Ana Catarina Sousa

Elisa Sousa

Rui Boaventura

Conselho Científico:

André Teixeira (Universidade Nova de Lisboa)

Carlos Fabião (Universidade de Lisboa)

Catarina Viegas (Universidade de Lisboa)

Gloria Mora (Universidad Autónoma de Madrid)

Grégor Marchand (Centre National de la Recherche Scientifique)

João Pedro Bernardes (Universidade do Algarve)

José Remesal (Universidade de Barcelona)

Leonor Rocha (Universidade de Évora)

Manuela Martins (Universidade do Minho)

Maria Barroso Gonçalves (Instituto Superior de Ciências do Trabalho e da Empresa)

Mariana Diniz (Universidade de Lisboa)

Raquel Vilaça (Universidade de Coimbra)

Xavier Terradas Battle (Consejo Superior de Investigaciones Científicas)

Secretariado: André Pereira

Capa: André Pereira sobre Báculo do Sobral do Martim Afonso (desenho de Marco Andrade).

Paginação: Elisa Sousa

Impressão: Europress

Data de impressão: Novembro de 2017

Edição impressa (preto e branco)

200 exemplares

Edição digital (a cores)

www.ophiussa.letras.ulisboa.pt

ISSN: $1645-653 X$

Depósito legal: 190404/03

Copyright $\odot 2017$, os autores

Edição:

UNIARQ - Centro de Arqueologia da Universidade de Lisboa

Faculdade de Letras de Lisboa

1600-214 - Lisboa

www.uniarq.net - www.ophiussa.letras.ulisboa.pt - uniarq@letras.ulisboa.pt

O cumprimento do acordo ortográfico de 1990 foi opção de cada autor. 


\section{ESTUDOS ARQUEOLÓGICOS DE OEIRAS, 22 VOLUMES E A CONTAR}

\section{VICTOR S. GONÇALVES*}

A publicação do vigésimo segundo volume de Estudos Arqueológicos de Oeiras (na verdade o vigésimo terceiro, uma vez que há um volume sem número em 1994) é, ao mesmo tempo, uma garantia e um motivo de regozijo. Uma garantia porque permite criar esperanças sobre a continuidade das publicações arqueológicas em Portugal. Um motivo de regozijo porque o feito de José Leite de Vasconcellos pode ser igualado e, assim se deseja, ultrapassado. Ao publicar os 30 volumes da primeira série de $O$ Archeologo Português o sábio do Restelo marcava a solidez de um projecto e a própria determinação, que sempre foi a dele, de garantir um abrigo para autores perdidos, jovens e menos jovens. E para ele próprio. O sábio do Funchalinho segue-lhe as pisadas...

João Luís Cardoso começou a série dos EAO e continuou, nem sempre com facilidades, uma missão similar à de Leite de Vasconcellos. Que maior elogio se lhe poderia fazer?

Tudo se passa como se os primeiros seis volumes dos EAO fossem um ensaio do que poderia vir a ser a série. Conscientemente, ou por simples acaso, este enumerar de possibilidades - volumes monográficos, reedições de títulos desaparecidos há muito, Actas de Congressos, Revistas científicas em edição standard, configura um projecto que se tornou (felizmente) quase uma obsessão para o seu motor. Enumeradas as possibilidades, a execução segue o curso natural dos acontecimentos.

Claro que um sítio constitui a espinha dorsal desta série. Liceia é, pela sua importância intrínseca e pela ligação efectiva (e afectiva) que João Luís Cardoso criou com ele, objecto central de um regresso mais ou menos regular. E o percurso, se pode parecer sinuoso, corresponde a uma busca de informação pluridisciplinar que constitui um exemplo raro em Portugal. Vila Nova de S. Pedro foi objecto de estudos incessantes por Afonso do Paço, mas as perspectivas são sempre as mesmas. Para Liceia, João Luís Cardoso vai usando os investigadores disponíveis para completar ângulos de visão, desde a fauna à pedra polida, desde a cerâmica decorada ao marfim. Liceia e as suas muralhas estão presentes até no logótipo, um pouco retro, que todos os volumes mantêm na capa, associando-se à folha de acácia e à folha de crucífera, ícones do Calcolítico das penínsulas de Lisboa e Setúbal.
E, naturalmente, a cronologia absoluta.

Todos sabemos (ainda que alguns procurem esquecer) que sem datas não há História e a chuva de datações para Liceia resolve parcialmente esta questão. Em 2007, ao divulgar, em O Arqueólogo Português, novas datações para a Rotura (Gonçalves e Sousa, 2007: 247, 251...), usámos da maior precaução, afastando os dados cronológicos obtidos através de conchas ou de madeira "suspeita». Ainda assim, o que restou impressiona através do número de datações disponíveis e da sua homogeneidade. Assim fosse com outros sítios e respirávamos certamente melhor...

\section{LER OS EAO}

Para uma leitura global dos EAO ébom fazermos uma referência aos seus conteúdos, procurando uma

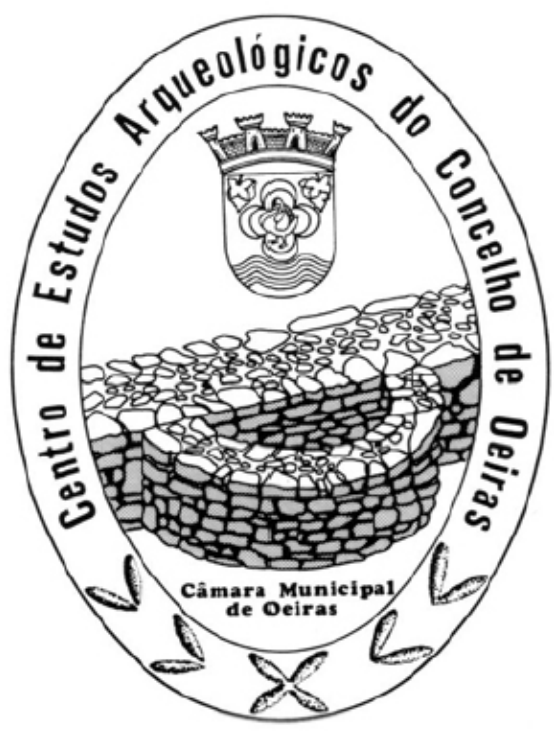

Fig. 1 - O Logótipo inicial dos EAO, ainda hoje presente, sem alterações, no volume 22.

lógica, que, no entanto, está longe de ser necessária. Esta é, naturalmente, uma perspectiva subjectiva. Notas de leitura e não uma análise crítica.

\subsection{A FASE DO ARRANQUE (VOLUMES 1 A 6)}

\section{Volume 1 (1991), reedição de livro}

Trata-se de um justo volume de homenagem a Carlos Ribeiro (1813-1882) não apenas publicado em fac-simile mas objecto de 200 comentários marginais da responsabilidade do editor. Pelo tipo de 
informação recolhida e divulgada, é um trabalho de grande utilidade, com detalhes por vezes menores, mas de algum modo úteis sobre as circunstâncias de vida do grande geólogo.

\section{Volume 2 (1991), livro}

J. L. Cardoso, Santinho Cunha, Aguiar, O Homem pré-histórico no concelho de Oeiras. Estudos de antropologia física, é um trabalho que reúne dados muito interessantes sobre antropologia física, com a vantagem de um dos co-autores ser um arqueólogo, o que não acontece com vários grupos de antropólogos físicos, com a obsessão da «patologia forense». Acredito que resultados exclusivamente patológicos sejam oportunos (quando não são oportunistas), mas o conhecimento dos contextos é indispensável até mesmo (e sobretudo) em leituras comparativas. Para mim, a História sempre foi Tempo e Contextos.

\section{Volume 3 (1992), livro}

J. L. Cardoso, associado a Georges Zbyszewski e Conceição André, publica aqui $O$ Paleolítico do complexo basáltico de Lisboa, um trabalho monumental num meio ainda hoje cheio de silêncios e suspiros paleolíticos que João Zilhão faria contrastar, sobretudo no caso do Paleolítico médio e superior.

\section{Volume 4 (1993), livro}

Cardoso (João Luís) e Cardoso (Guilherme) apresentam a Carta arqueológica de Oeiras. Trabalho útil, ainda que se justificassem considerações mais extensas sobre a diacronia ilustrada.

\section{Volume sem número (1994), livro}

Um bem vindo balanço de dez anos de escavações em Liceia (1983-1993), que bem poderia ter sido o volume $5 . .$. , mas que foi considerado apenas um «número especial». E um número especial é, com efeito, tornando evidentes os contributos de uma escavação persistente de um sítio importante. Uma situação de algum modo exemplar. Quantos monumentos e sítios de grande importância foram escavados em Portugal sem publicação subsequente ou mesmo abandonados, sem que se separasse o sumo da casca (como setubalense, sei bem que a casca - da laranja - também se come, mas a diferença conta).

\section{Volume 5 (1995), Revista}

Dos 11 textos, J. L. C. está em todos, em 10 como primeiro autor. De novo, Liceia, mas também Penha Verde, Montes Claros...

\section{Volume 6 (1996), Revista}

Neste volume, dos 19 artigos que o integram, J. L. C. está em 15 como autor principal e em 2 como segundo autor. Esta produtividade não é rara nos EAO e abrange textos de balanço (o Neolítico antigo da região de Lisboa, o campaniforme de Sintra, o Ferro 2), a publicação micro-monográfica de artefactos e sítios (Carnaxide, Liceia, Tituaria, Senhora da Luz...). Um artigo muito importante, injustamente esquecido, de Miguel Kunst, reabre a discussão e a periodização do Calcolítico a partir da cerâmica decorada do Zambujal.

\subsection{OS ANOS DE CONFIRMAÇÃO (VOLUMES 7 A 16)}

\section{Volume 7 (1997-1998), Revista}

Dos 11 artigos de fundo, J. L. C. assina 10, oito em solo, um em primeiro, com colaboração, e um em segundo. O falecido Justino Mendes de Almeida (1924-2012) assina sozinho um texto, como sempre inteligentemente escrito.

Liceia, como se compreende, mais uma vez domina.

\section{Volume 8 (1999-2000), Revista}

Não é um volume típico, mas inclui uma curta bio-bibliografia de Georges Zbyszewski (1909-1999), investigador muito apreciado pelo seu trabalho e também pela gentileza de trato, rara em Portugal entre arqueólogos. A preocupação de João Luís Cardoso em homenagear os seus antecessores, constante ao longo de toda a sua vida, faz com que se incluam neste volume notas biográficas ou a propósito de personagens tão diversas como o Marquês de Pombal, José Leite de Vasconcellos, Carlos Ribeiro ou Nery Delgado. A página 489, um texto auto-biográfico intitulado «Vinte e cinco anos de trabalhos arqueológicos» onde o editor apresenta e comenta o seu percurso. Muito importante é também uma análise da história da investigação dos concheiros de Muge (p. 83-240), profusamente documentada.

\section{Volume 9 (2000), livro}

Abrindo inesperadamente com um belo soneto de Antero de Quental, Sítios, pedras e homens: trinta anos de Arqueologia em Oeiras, é um volume surpreendente, para quem leu os anteriores volumes dos EAO. Sintoniza bem com a Carta Arqueológica e, de algum modo, com as sínteses publicadas sobre Liceia. 


\section{Volume 10 (2002), Revista}

A nível da história da Arqueologia, para além da oração produzida na Academia Portuguesa da História a propósito de Manuel Farinha dos Santos, texto com alguma utilidade uma vez que pouco se sabia sobre a vida científica daquele antigo assistente da Faculdade de Letras de Lisboa, protegido de Manuel Heleno, há também correspondência de Abel Viana e Veiga Ferreira. É um volume com composição diversificada, onde predominam textos sobre arqueofaunas, de grande interesse, focando mamutes, e sobretudo as recolhas de Liceia. É também neste volume que se publica um dos mais notáveis artefactos votivos de calcário recolhidos na Estremadura portuguesa, mais propriamente no dolmen de Casaínhos.

\section{Volume 11 (2003), Revista}

Nunca compreendi bem a organização de este volume, que abre aliás com um estudo interessante, de Maria Leonor Machado de Sousa, sobre os viajantes estrangeiros em Portugal nos sécs. 18 e 19. É um tema pertinente, mas poderia ter sido colocado em fim de volume, que ganharia assim uma sequência diacrónica. Mas este é também o volume em que é publicado um sítio importantíssimo, numa perspectiva de recuperação monotemática dos dados arqueológicos de trabalhos anteriores. A Gruta do Correio Mor é aqui objecto de uma metodologia da maior utilidade para os arqueólogos que não tinham facilidade em aceder a colecções vastas, mas de grande importância. 61 Figuras ilustram exaustivamente este reinventário. À maneira do que J. L. C. tinha feito para a Lapa do Bugio, na Setúbal Arqueológica 9-10. E, para além dos materiais calcolíticos, são ainda referidos artefactos das Idades do Bronze, do Ferro e mesmo alguns medievais.

\section{Volume 12 (2004), livro}

Do $4^{\circ}$ milénio a acabar até à invasão romana, no séc. 2 antes da nossa Era.

J. L. C. é autor de trabalhos ambiciosos, como a Pré-História de Portugal (2002 e 2007). Aqui, discorre sobre o tempo longo, numa diacronia que não é curta, mas numa região delimitada, a «Baixa Estremadura». Modestamente, chama-lhe «um ensaio de História Regional», mas, na verdade, é maior que isso. A maioria dos grandes temas está presente e se alguns variam de importância outros constituem leituras difíceis e parcialmente por fazer (Idades do Bronze e do Ferro).

\section{Volume 13 (2006), Revista}

A publicação da correspondência de Luís Fontes (1892-1960) garante a continuidade da recuperação da memória, patente em muitos volumes dos EAO. E o monumento megalítico do Monte Serves, com North e o actualmente falecido Rui Boaventura (19712016) continua a política correcta de republicação de dados antigos, mesmo aqueles que forneceram escassa informação, como este.

\section{Volume 14 (2006), Revista}

Estácio da Veiga e a Arqueologia é outro regresso ao passado da nossa ciência, desta vez ao tempo dos «Pais fundadores». Personagem mais complexa do que poderia parecer, Estácio da Veiga bem justifica toda a atenção possível, até mesmo como vítima de Leite de Vasconcellos, na altura no topo da cadeia alimentar... Mas, falando de comida, as cerâmicas decoradas de Liceia são o prato principal de este volume.

Volume 15 (2007), híbrido (Revista e Actas de Colóquio).

Sub-intitulado A Arqueologia portuguesa e o espaço europeu. Balanços e perspectivas.

Trata-se das Actas de um colóquio que decorreu na Sociedade de Geografia em 30 de Outubro de 2007.

Alguns artigos nada têm que ver com a temática (o meu, por exemplo, mas também vários outros) e a empáfia de alguns é, no mínimo, divertida.

Volume 16 (2008), só teoricamente, um número da Revista, na verdade exclusivamente uma Homenagem a Octávio da Veiga Ferreira

Bem e mal, como acontece muitas vezes, o Eng $^{\circ}$ Veiga Ferreira marcou a Arqueologia portuguesa do séc. 20. De forma indelével. Mas em Portugal é hábito esquecer intencionalmente as pessoas de que se não gosta. Às vezes, é um caso de higiene e ainda bem, mas em outras situações a realidade é outra, traduzindo de algum modo a mesquinhez nacional.

João Luís Cardoso foi realmente amigo e admirador de Veiga Ferreira, enquanto eu pessoalmente preferia Georges Zbyszewski, pela inteligência e boas maneiras. Mas é forçoso reconhecer que Veiga Ferreira, a quem faltava a formação teórica, era um homem de terreno, um marine da Arqueologia. E conhecia excelentemente os materiais arqueológicos com que tratava. Esta homenagem, traduzida num volume de capa dura, numerosas ilustrações (várias a cores) e um total 
de 751 páginas, é uma sincera homenagem de um arqueólogo que não esquece os seus amigos. Por isso, bem haja, como escreve o outro.

\subsection{VELOCIDADE DE CRUZEIRO (VOLUMES 17 E SEGUINTES)}

Volume 17 (2009), Revista, número comemorativo do $20^{\circ}$ aniversário do CEA de Oeiras

718 páginas num volume de capa dura assinalam uma reunião considerável de arqueólogos muito diferentes entre si. 0 \# 17 é definitivamente uma Revista, no sentido técnico do termo, mas é também um repositório de questões-chave, particularmente no que à Pré-História e à Idade do Bronze diz respeito. Alguma desordem diacrónica, mas mínima.

\section{Volume 18 (2010-2011), Revista}

Um volume graficamente de grande qualidade (Capa dura, imagens parcialmente a cores...) só peca pela desordem diacrónica (Calcolítico - Bronze Ferro - Romano - Idade moderna - Megalitismo Calcolítico - Romano - Neolítico - Calcolítico... uma sequência incompreensível. Felizmente, termina em bem, com uma série de textos de J. L. C. sobre história da Arqueologia, Francisco Jordá Cerdá, Joaquim Fontes, Mendes Corrêa (que tão insuficientemente estudado tem sido).

A salientar o importante estudo de Miguel Kunst e Nina Lutz sobre a cronologia absoluta do Zambujal, a republicação da Penha Verde e a cronologia absoluta do Outeiro Redondo, ainda que esta apresente um leque muito complexo de problemas e justifique outro espaço de discussão.

\section{Volume 19 (2012), Actas de Colóquio}

Uma sequência de artigos desordenada e mesmo caótica, de publicação provavelmente difícil não fora a disponibilização dos EAO.

\section{Volume 20 (2013), híbrido (Revista e Actas de Colóquio)}

Dedicado a Carlos Ribeiro (1813-1882)

Mais um volume graficamente de qualidade (Capa dura, imagens parcialmente a cores...). Parte de um Colóquio organizado pela CMO em colaboração com a Academia das Ciências de Lisboa, enriquecendo-se com outras contribuições. Como acontece frequentemente nestas situações, há um pouco de tudo, do melhor ao pior. É um volume de tipologia atípica, mais uma miscelânea de estudos que as Actas de um Congresso, em que os dados são tratados sem uma correlação específica, à excepção dos que têm como objecto Carlos Ribeiro.

\section{Volume 21 (2014), Revista}

Tenho dificuldade em sumariar de forma crítica este volume, por conter um texto que me irrita, versão de um outro publicado de origem noutra língua. Eventualmente oportuno para desmascarar uma leitura demencial de um artefacto típico da Pré-História de Portugal, mas com uma construção evidenciando insuficiências de leituras, quando não «inspirações» com origens não explicitamente referidas. E outro, assinado por mim, Marco Andrade e André Pereira, que analisa outra situação, muito particular, sobre leituras regionais do mesmo fenómeno. Mas este volume 21 acrescenta à bibliografia arqueológica portuguesa textos muito oportunos, como os dedicados a Moita da Ladra, ao campaniforme, a Chões de Alpompé, Outurela e... Abel Viana...

\section{Volume 22 (2015), Revista}

Pude consultar o índice do volume 22 e fiquei alerta para os textos de João Luís Cardoso «Na Estremadura do Neolítico Antigo ao Neolítico Final: contributos de um percurso pessoal»; de João Luís Cardoso, Ana Catarina Sousa e Maria da Conceição André, «O povoado do Carrascal (Oeiras). Estudo das ocupações do Neolítico Final e do Calcolítico»; de Cláudia Costa e Francisco Rosa Correia, «A componente animal no Calcolítico Pleno da Estremadura portuguesa: o conjunto de fauna do Alto de Santo Antão (Óbidos)» e ainda de Ana Margarida Arruda e João Luís Cardos, «A necrópole da Idade do Ferro de Vale da Palha (Calhariz, Sesimbra)», este último exclusivamente pelo meu recente, ainda que limitado, regresso a Setúbal...

\section{CONSIDERAÇÕES FINAIS}

Aideia que persisteéassim a de uma construção monumental, que arranca experimentalmente, vai crescendo e regista, por vezes, a desordem que se reflecte na produção actual da Arqueologia portuguesa. Na verdade, os EAO, na sua tipologia, são coisas diferentes de volume para volume: uns são livros autênticos (7), outros Revistas tradicionais (13), outros Actas de Colóquios (1), enriquecidas ou não com estudos adicionais, formando-se assim volumes híbridos (2). O que não é forçosamente mau, reflectindo as características actuais da Arqueologia 
portuguesa, a nível de publicações sempre muito irregular. Preencher um volume dos EAO com textos homogéneos não é tarefa fácil, o que explica também a densidade das contribuições produzidas pelo próprio editor, cuja omnipresença impressiona e deve ser registada com apreço.

Lisboa, Outono de 2016

\section{REFERÊNCIAS BIBLIOGRÁFICAS}

CARDOSO, J. L. (1992) - A Lapa do Bugio. Setúbal Arqueológica 9-10: 89-225.

CARDOSO, J. L. (1997) - O povoado de Leceia sentinela do Tejo no terceiro milénio antes de Cristo. Lisboa/ Oeiras.
CARDOSO, J. L. (2002) - Pré-História de Portugal. Lisboa.

CARDOSO, J. L. (2007) - Pré-História de Portugal. Lisboa (com CD-Rom de figuras).

GONÇALVES, V. S. (1995) - Sítios, "Horizontes» $e$ Artefactos. Leituras críticas de realidades perdidas. Cascais, $1^{\text {a }}$ edição.

GONÇALVES, V. S. (2003) - Sítios, "Horizontes»e artefactos. Estudos sobre o $3 .^{\circ}$ milénio no Centro e Sul

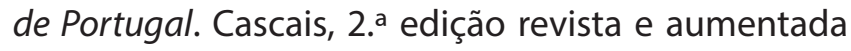
com dois novos textos.

GONÇALVES, V. S. - SOUSA, A. C. (2007) - Algumas breves reflexões sobre quatro datas $14 C$ para o Castro da Rotura, no contexto de $3 .^{\circ}$ milénio a.n.e. nas Penínsulas de Lisboa e Setúbal. O Arqueólogo Português IV-24: 233-266.

\footnotetext{
* - Universidade de Lisboa, Faculdade de Letras, Uniarq - Centro de Arqueologia da Universidade de Lisboa / WAPS - Workgroup on Ancient Peasant Societies; vsg@campus.ul.pt
} 


\section{OPHIUSSA}

\section{POLÍTICA EDITORIAL}

A Ophiussa - Revista do Centro de Arqueologia da Universidade de Lisboa foi iniciada sob a direcção de Victor S. Gonçalves em 1996, tendo sido editado o volume 0. O volume 1 (2017) é uma edição impressa e digital da UNIARQ - Centro de Arqueologia da Universidade de Lisboa.

O principal objectivo desta revista é a publicação e divulgação de trabalhos com manifesto interesse, qualidade e rigor científico sobre temas de Pré-História e Arqueologia, sobretudo do território europeu e da bacia do Mediterrâneo.

A Ophiussa - Revista do Centro de Arqueologia da Universidade de Lisboa publicará um volume anual. A partir de 2018, os artigos submetidos serão sujeitos a um processo de avaliação por parte de revisores externos (peer review). O período de submissão de trabalhos decorrerá sempre no primeiro trimestre e a edição ocorrerá no último trimestre de cada ano.

A revista divide-se em duas secções: artigos científicos e recensões bibliográficas. Excepcionalmente poderão ser aceites textos de carácter introdutório, no âmbito de homenagens ou divulgações específicas, que não serão submetidos à avaliação por pares. Isentas desta avaliação estão também as recensões bibliográficas.

Todas as submissões serão avaliadas, em primeira instância, pela Coordenação Editorial, no que respeita ao seu conteúdo formal e à sua adequação face à política editorial e às normas de edição da revista. Os trabalhos que cumprirem estes requisitos serão posteriormente submetidos a um processo de avaliação por pares cega / blind peer review (mínimo de dois revisores). O Conselho Científico, constituído pela direcção da UNIARQ e por investigadores externos, seleccionará os revisores e acompanhará o processo de edição.

Esta etapa será concretizada por investigadores externos qualificados, sendo os respectivos pareceres entregues num período não superior a três meses. Os revisores procederão à avaliação de forma objectiva, tendo em vista a qualidade do conteúdo da revista; as suas críticas, sugestões e comentários serão, na medida do possível, construtivos, respeitando as capacidades intelectuais do(s) autor(es). Após a recepção dos pareceres, o(s) autor(es) tem um prazo máximo de um mês para proceder às alterações oportunas e reenviar o trabalho.

A aceitação ou recusa de artigos terá como únicos factores de ponderação a sua originalidade e qualidade científica. $\mathrm{O}$ processo de revisão é confidencial, estando assegurado o anonimato dos avaliadores e dos autores dos trabalhos, neste último caso até à data da sua publicação.

Os trabalhos só serão aceites para publicação a partir do momento em que se conclua o processo da revisão por pares. Os textos que não forem aceites serão devolvidos aos seus autores.

O conteúdo dos trabalhos é da inteira responsabilidade do(s) autor(es) e não expressa a posição ou opinião do Conselho Científico ou da Coordenação Editorial.

As ilustrações que não sejam do(s) autor(es) devem indicar a sua procedência. O Conselho Científico e a Coordenação Editorial assumem que os autores solicitaram e receberam autorização para a reprodução dessas ilustrações, e, como tal, rejeitam a responsabilidade do uso não autorizado das ilustrações e das consequências legais por infracção de direitos de propriedade intelectual.

A publicação de textos na Ophiussa - Revista do Centro de Arqueologia da Universidade de Lisboa não implica o pagamento de qualquer taxa nem dá direito a qualquer remuneração económica.

Os textos propostos para publicação devem ser inéditos e não deverão ter sido submetidos a qualquer outra revista ou edição electrónica. Aceitam-se trabalhos redigidos em português, inglês, espanhol, italiano e francês.

Esta edição disponibiliza de imediato e gratuitamente a totalidade dos seus conteúdos, em acesso aberto, de forma a promover, globalmente, a circulação e intercâmbio dos resultados da investigação científica e do conhecimento.

Esta publicação dispõe de uma versão impressa, a preto e branco, com uma tiragem limitada, que será distribuída gratuitamente pelas bibliotecas e instituições mais relevantes internacionalmente, e intercambiada com publicações periódicas da mesma especialidade, que serão integradas na Biblioteca da Faculdade de Letras da Universidade de Lisboa. Conta, paralelamente, com uma versão digital, a cores, disponibilizada no endereço www.ophiussa.letras.ulisboa.pt, onde se pode consultar a totalidade da edição.

Para mais informações contactar: uniarq@letras.ulisboa.pt 


\section{OPHIUSSA}

\section{EDITORIAL POLICY}

Ophiussa - Revista do Centro de Arqueologia da Universidade de Lisboa started under the direction of Victor S. Gonçalves in 1996, with the edition of volume 0. Volume 1 (2017) is a printed and digital edition of UNIARQ - Centro de Arqueologia da Universidade de Lisboa.

The main objective of this journal is the publication and dissemination of papers of interest, quality and scientific rigor concerning Prehistory and Archeology, mostly from Europe and the Mediterranean basin.

Ophiussa - Revista do Centro de Arqueologia da Universidade de Lisboa will publish an annual volume. From 2018, submitted articles will be subject to a peer-review evaluation process. The submission period will always occur in the first quarter of each year and the edition will occur in the last quarter.

The journal is divided into two sections: scientific articles and bibliographic reviews. Exceptionally, texts of an introductory nature may be accepted, in the context of specific tributes or divulgations, which will not be submitted to peerreview evaluation. Exemptions from this evaluation are also the bibliographic reviews.

All submissions will be considered, in the first instance, by the Editorial Board, regarding its formal content and adequacy in face of the editorial policy and the journal's editing standards. Papers that meet these requirements will subsequently be submitted to a blind peer-review process (minimum of two reviewers). The Scientific Council, constituted by the directors of UNIARQ and external researchers, will select the peer-reviewers and follow the editing process.

This stage will be carried out by qualified external researchers, and their feedback will be delivered within a period of no more than two months. The reviewers will carry out the evaluation in an objective manner, in view of the quality and content of the journal; their criticisms, suggestions and comments will be, as far as possible, constructive, respecting the intellectual abilities of the author (s). After receiving the feedback, the author(s) has a maximum period of one month to make the necessary changes and resubmit the work.

Acceptance or refusal of articles will have as sole factors of consideration their originality and scientific quality. The review process is confidential, with the anonymity of the evaluators and authors of the works being ensured, in the latter case up to the date of its publication.

Papers will only be accepted for publication as soon as the peer review process is completed. Texts that are not accepted will be returned to their authors.

The content of the works is entirely the responsibility of the author(s) and does not express the position or opinion of the Scientific Council or Editorial Board.

Illustrations that are not from the author(s) must indicate their origin. The Scientific Council and Editorial Board assume that the authors have requested and received permission to reproduce these illustrations and, as such, reject the responsibility for the unauthorized use of the illustrations and legal consequences for infringement of intellectual property rights.

The publication of texts in Ophiussa - Revista do Centro de Arqueologia da Universidade de Lisboa does not imply the payment of any fee nor does it entitle to any economic remuneration.

Texts proposed for publication must be unpublished and should not have been submitted to any other journal or electronic edition. Works written in Portuguese, English, Spanish, Italian and French are accepted.

This edition immediately and freely provides all of its content, in open access, in order to promote global circulation and exchange of scientific research and knowledge.

This publication has a limited printed edition in black and white, which will be distributed free of charge by the most relevant international libraries and institutions, and exchanged with periodicals of the same specialty, which will be integrated in the Library of Faculdade de Letras of Universidade de Lisboa. It also has a digital version, in color, available at address www. ophiussa.letras.ulisboa.pt, where one can consult the entire edition.

For more information contact: uniarq@letras.ulisboa.pt 


\section{OPHIUSSA}

REVISTA DO CENTRO DE ARQUEOLOGIA DA UNIVERSIDADE DE LISBOA

\section{1 - 2017}

\section{ÍNDICE}

VICTOR S. GONÇALVES - Ophiussa regressa, em digital e, logo de seguida, em papel .... MARCO ANTÓNIO ANDRADE - O sítio pré-histórico do Sobral do Martim Afonso (Sätuatterra de Magos, Portugal): um curioso contexto do Neolítico Final / Calcolítico na marégentesquerda do Baixo Tejo

ANA CATARINA SOUSA - JORGE LOPES - O sítio do Moinho do Custódio (tarruda dos vinthost.

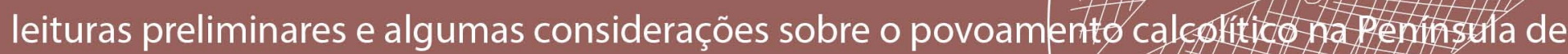
Lisboa

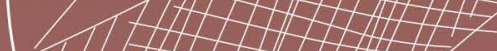
51

PEDRO ALBUQUERQUE - O Guadiana como fronteira? Notas para un profecto-de investigação

ANA MARGARIDA ARRUDA - ELISA DE SOUSA - JOÃO PIMENTA - RUI SOARES - HENRIQU MENDES - Fenícios e indígenas em contacto no Estuário do Tejo

ELISA DE SOUSA - Algumas reflexões sobre a fase tardia da Idade do Ferro no Ocidente Atlântico

CARLOS PEREIRA - Produção e comércio de lucernas durante a Antiguidade Tardia: génese e evolução das lucernas tardo-antigas de produção africana

JACINTA BUGALHÃO - O papel da mulher na Arqueologia Portuguesa

RUI BOAVENTURA - VERA.LEISNER@PORTUGAL.PT

RECENSÕES BIBLIOGRÁFICAS - Estudos Arqueológicos de Oeiras, 22 volumes e a contar... (VICTOR S. GONÇALVES) 